\title{
Modified (A)dS Schwarzschild black holes in Rainbow spacetime
}

\author{
Huarun Li, ${ }^{*} \mathrm{Yi}$ Ling, ${ }^{\dagger}$ and $\mathrm{Xin} \mathrm{Han}^{\ddagger}$ \\ Center for Gravity and Relativistic Astrophysics, Department of Physics, \\ Nanchang University, Nanchang 330031, China
}

\begin{abstract}
A modified (Anti-)de Sitter Schwarzschild black hole solution is presented in the framework of rainbow gravity with a cosmological constant. Its thermodynamical properties are investigated. In general the temperature of modified black holes is dependent on the energy of probes which take the measurement. However, a notion of intrinsic temperature can be introduced by identifying these probes with radiation particles emitted from black holes. It is interesting to find that the Hawking temperature of this sort of black holes can be reproduced by employing the extended uncertainty principle and modified dispersion relations to the ordinary (A)dS Schwarzschild black holes.
\end{abstract}

*Electronic address: alloys91@sohu.com

${ }^{\dagger}$ Electronic address: yling@ncu.edu.cn

${ }^{\ddagger}$ Electronic address: bifrostx@gmail.com 


\section{INTRODUCTION}

Doubly Special Relativity (DSR) has gradually been viewed as an effective theory to describe physics phenomena at extremely high energy level when the semi-classical effects of quantum gravity is taken into account $[1,2,3,4,5,6,7,8]$. In this framework the Lorentz symmetry may be preserved or deformed. One of its key ingredients is that the usual energymomentum or dispersion relation may be modified with corrections in the order of Planck length $l_{p}=\sqrt{8 \pi G} \sim 1 / M_{p}$

$$
E^{2} f^{2}\left(l_{p} E\right)-P^{2} g^{2}\left(l_{p} E\right)=m^{2},
$$

where $f(E)$ and $g(E)$ are two general functions of energy with a constraint that they approach to unit as the energy of particles is much less than the Planck energy $M_{p}$. Modified dispersion relations (MDR) and its implications to physics have been greatly investigated in recent years[1,9]. They may be responsible for threshold anomalies of ultra high energy cosmic rays and Gamma ray burst[10, 11, 12, 13, 14, 15, 16], contribute corrections to the entropy of black holes [17, 18], provide alternatives to inflationary cosmology[19, 20], and disclose the nature of dark energy[21].

Recently DSR has been generalized to incorporate the effects of gravity, leading to a deformed formalism of general relativity, which is always named as rainbow gravity because the metric of the background detected by any probe is not fixed, but depends on the energy of the probe[22]. Specifically, given a modified dispersion relation as in Eq.(1), the usual flat metric is replaced by a one-parameter family of rainbow metric

$$
d s^{2}=-\frac{1}{f^{2}(E)} d t^{2}+\frac{1}{g^{2}(E)} d x^{2},
$$

such that the contraction between momentum and infinitesimal displacement

$$
d x^{\mu} p_{\mu}=d t E+d x^{i} p_{i}
$$

be an invariant quantity. This strategy overcomes the difficulty of defining the position space conjugate to momentum space arising in $D S R$ where the Lorentz symmetry is accomplished by nonlinear transformations in momentum space[48]. When the curvature of spacetime is taken into account, a deformed equivalence principle of general relativity is proposed, requiring that the free falling observers who make measurements with energy $E$ will observe the same laws of physics as in doubly special relativity. As a consequence, in rainbow 
gravity the original Einstein field equation is replaced by a one-parameter family of modified equations,

$$
G_{\mu \nu}(E)=8 \pi G(E) T_{\mu \nu}(E)+g_{\mu \nu}(E) \Lambda(E)
$$

where Newton's constant as well as the cosmological constant is conjectured to be energy dependent as one expects from the viewpoint of renormalization group theory[24, 25].

This formalism has received a lot of attention and some relevant work can be found in Ref.[26, 27, 28, 29, 30, 31, 32, 33, 34, 35, 36, 37]. In particular in [30] one of our authors with other collaborators studied the thermodynamics of modified Schwarzschild black holes appearing in rainbow gravity. Although the temperature of such kind of black holes is also energy dependent, a notion of intrinsic temperature was introduced by identifying probes with those photons surrounding the vicinity of black hole horizon.

In this short report we intend to extend this strategy to rainbow gravity with a cosmological constant. We firstly show in section two that there exists a modified (A)dS Schwarzschild black hole solution to the modified vacuum field equation, then turn to investigate the thermodynamics of this sort of black holes in section three. Given a specific modified dispersion relation, the intrinsic temperature can be obtained. Comparing it with the one obtained in Ref.[38], where the impact of modified dispersion relation on ordinary (A)dS-Schwarzschild black holes is investigated, we find that they are exactly the same such that the proposal of deformed equivalence principle is testified in this context.

\section{THE MODIFIED (A)DS SCHWARZSCHILD SOLUTION}

In this section we demonstrate a spherically symmetric solution to the modified field equations (4) with non-zero cosmological constant. In the absence of matter, it reduces to $G_{\mu \nu}(E)=\Lambda(E) g_{\mu \nu}$, where $\Lambda(E)= \pm 3 / L^{2}(E)$ is an energy-dependent cosmological constant, where "+" sign and "-" sign correspond to dS and AdS cases respectively. For explicitness let us focus on the AdS black holes in following discussions but all derivations can be applied to the de-Sitter case directly. Given a general modified dispersion relation as shown in (1), we may write the most general form for a spherically symmetric metric in energy independent coordinates as

$$
d s^{2}=-\frac{e^{2 a(r)}}{f^{2}(E)} d \tau^{2}+\frac{e^{2 b(r)}}{g^{2}(E)} d r^{2}+\frac{r^{2}}{g^{2}(E)}\left(d \theta^{2}+\sin ^{2} \theta d \phi^{2}\right),
$$

where $a(r)$ and $b(r)$ are two functions we intend to solve for. They may be energy-dependent. 
Now it is straightforward to obtain the non-vanishing components of the connection as follows

$$
\begin{array}{lll}
\Gamma_{10}^{0}=\Gamma_{01}^{0}=a^{\prime}, & \Gamma_{00}^{1}=g^{2}(E) f^{-2}(E) e^{2(a-b)} a^{\prime}, & \Gamma_{11}^{1}=b^{\prime}, \\
\Gamma_{22}^{1}=-r e^{-2 b}, & \Gamma_{33}^{1}=-r \sin ^{2} \theta e^{-2 b}, & \Gamma_{12}^{2}=\Gamma_{21}^{2}=r^{-1}, \\
\Gamma_{13}^{3}=\Gamma_{31}^{3}=r^{-1}, & \Gamma_{33}^{2}=-\sin \theta \cos \theta, & \Gamma_{23}^{3}=\Gamma_{32}^{3}=\cot \theta,
\end{array}
$$

where a prime sign denotes the derivative with respect to the radius $r$. Thus the nonvanishing Ricci tensor components are

$$
\begin{aligned}
& R_{00}=\frac{g^{2}(E)}{f^{2}(E)} e^{2(a-b)}\left[a^{\prime \prime}-a^{\prime} b^{\prime}+a^{2}+2 r^{-1} a^{\prime}\right], \\
& R_{11}=-\left[a^{\prime \prime}-a^{\prime} b^{\prime}+a^{\prime 2}-2 r^{-1} b^{\prime}\right], \\
& R_{22}=-e^{-2 b}\left[1+r\left(a^{\prime}-b^{\prime}\right)\right]+1, \\
& R_{33}=\left\{-e^{-2 b}\left[1+r\left(a^{\prime}-b^{\prime}\right)\right]+1\right\} \sin ^{2} \theta,
\end{aligned}
$$

and the Ricci scalar is

$$
R=2 g^{2}(E) r^{-2}+2 g^{2}(E) e^{-2 b}\left[\left(-a^{\prime \prime}+a^{\prime} b^{\prime}-a^{\prime 2}\right)-r^{-2}-2 r^{-1}\left(a^{\prime}-b^{\prime}\right)\right] .
$$

Substituting all these terms into the AdS vacuum field equations gives rise to

$$
\begin{gathered}
-g^{2}(E) e^{-2 b}\left(2 r^{-1} b^{\prime}-r^{-2}\right)-g^{2}(E) r^{-2}=\frac{3}{L^{2}(E)}, \\
g^{2}(E) e^{-2 b}\left(2 r^{-1} a^{\prime}+r^{-2}\right)-g^{2}(E) r^{-2}=\frac{3}{L^{2}(E)}, \\
g^{2}(E) e^{-2 b}\left[a^{\prime \prime}-a^{\prime} b^{\prime}+a^{\prime 2}+r^{-1}\left(a^{\prime}-b^{\prime}\right)\right]=\frac{3}{L^{2}(E)} .
\end{gathered}
$$

From (9) and (10) we can obtain a relation between $a$ and $b$ as

$$
a=-b+\alpha
$$

with $\alpha$ being a constant. Furthermore we can obtain $e^{-2 b}$ from (9) and (10) as

$$
e^{-2 b}=1+c r^{-1}+g^{-2}(E) L^{-2}(E) r^{2},
$$

where $c$ is an integral constant.

As a result, the rainbow metric in eq.(5) becomes

$$
d s^{2}=-\frac{N^{2} e^{2 \alpha}}{f^{2}(E)} d \tau^{2}+\frac{1}{g^{2}(E) N^{2}} d r^{2}+\frac{r^{2}}{g^{2}(E)}\left(d \theta^{2}+\sin ^{2} \theta d \phi^{2}\right),
$$

where $N^{2}=1+c r^{-1}+g^{-2}(E) L^{-2}(E) r^{2}$. 
As usual we can define a new coordinate by $t=e^{\alpha} \tau$ such that the metric becomes

$$
d s^{2}=-\frac{N^{2}}{f^{2}(E)} d t^{2}+\frac{1}{g^{2}(E) N^{2}} d r^{2}+\frac{r^{2}}{g^{2}(E)}\left(d \theta^{2}+\sin ^{2} \theta d \phi^{2}\right) .
$$

Now the remaining task is to determine the constant $c$. Firstly we notice that as the cosmological constant vanishes, this solution should recover the modified Schwarzschild solution which has been obtained in [22]. This directly leads to $c=-2 G(0) M$. As a matter of fact we point out that this fixing implies that we have set the energy dependent Newton's constant as $G(E)=g^{-1}(E) G(0)$. When the cosmological constant is not zero, we argue that it is appropriate to set $\Lambda(E)=g^{2}(E) \Lambda(0)$ based on the following considerations. Given a rainbow metric as Eq.(5), we notice that in general the volume of a spatial region with fixed size $R$ is energy dependent as $V \sim g^{-3}(E) V_{0}$. It is reasonable to require that the total vacuum energy in this region is independent of the energy of free-falling probes, namely

$$
E_{\text {vac }} \sim \rho(E) V(E) \sim \frac{\Lambda(E)}{8 \pi G(E)} g^{-3}(E) V_{0} \sim \text { Const. }
$$

Once Newton's constant is set as $G(E)=g^{-1}(E) G(0)$, we find the condition above is satisfied if

$$
\Lambda(E)=g^{2}(E) \Lambda(0)= \pm \frac{3 g^{2}(E)}{L^{2}(0)} .
$$

Finally the rainbow metric for modified AdS Schwarzschild black holes takes the form

$$
d s^{2}=-\frac{N^{2}}{f^{2}(E)} d t^{2}+\frac{1}{g^{2}(E) N^{2}} d r^{2}+\frac{r^{2}}{g^{2}(E)}\left(d \theta^{2}+\sin ^{2} \theta d \phi^{2}\right),
$$

where $N^{2}=1-2 G(0) M r^{-1}+L^{-2}(0) r^{2}$.

It is manifest that at low energy limit, namely $E / M_{p} \rightarrow 0$, this solution becomes the conventional anti-de-Sitter Schwarzschild vacuum solution.

$$
d s^{2}=-N^{2} d t^{2}+\frac{1}{N^{2}} d r^{2}+r^{2}\left(d \theta^{2}+\sin ^{2} \theta d \phi^{2}\right) .
$$

In parallel the modified dS Schwarzschild black hole solution to the modified vacuum field equation can be easily obtained by considering $L^{2}(0) \longrightarrow-L^{2}(0)$ in $(18)$ and it can return to the ordinary dS Schwarzschild vacuum solution at the low energy limit.

In these solutions, the position of horizon is determined by

$$
2 G(0) M=r_{+}\left(1 \pm \frac{r_{+}^{2}}{L^{2}(0)}\right)
$$

where "+" sign and " - " sign correspond to AdS and dS black holes respectively. Thus, in general the area of the horizon is energy dependent as $A=\left(4 \pi r_{+}^{2}\right) / g^{2}(E)$. 


\section{THERMODYNAMICS WITH THE MODIFIED (A)DS SCHWARZSCHILD BLACK HOLES}

Now we turn to investigate the thermodynamics of the modified (A)dS Schwarzschild black holes. As pointed out in [30], in the framework of rainbow gravity, the temperature of the modified (A)dS Schwarzschild black holes (18) can be identified with the surface gravity $\kappa$ on the horizon, namely $T=\kappa /(2 \pi)$, where $\kappa$ is related to the metric by

$$
\kappa=-\frac{1}{2} \lim _{r \longrightarrow r_{+}} \sqrt{\frac{-g^{11}}{g^{00}}} \frac{\left(g^{00}\right)^{\prime}}{g^{00}} .
$$

Now it is straightforward to obtain the surface gravity for modified Schwarzschild (A)dS black holes as

$$
\kappa=\frac{g(E)}{2 f(E)}\left(\frac{2 G M}{r_{+}^{2}} \pm \frac{2 r_{+}}{L^{2}(0)}\right)=\frac{g(E)}{2 f(E)}\left(\frac{1}{r_{+}} \pm \frac{3 r_{+}}{L^{2}(0)}\right)
$$

where " +" sign and " - " sign correspond to AdS and dS black holes respectively. Thus, the temperature reads as

$$
T_{(A) d S}=\frac{\kappa}{2 \pi}=\frac{g(E)}{4 \pi f(E)}\left(\frac{1}{r_{+}} \pm \frac{3 r_{+}}{L^{2}(0)}\right) \equiv \frac{g(E)}{f(E)} T_{0(A) d S},
$$

where $T_{0(A) d S}$ is understood as the temperature for the ordinary $(\mathrm{A}) \mathrm{dS}$ black holes, i.e.

$$
T_{0(A) d S}=\frac{1}{4 \pi}\left(\frac{1}{r_{+}} \pm \frac{3 r_{+}}{L^{2}(0)}\right)
$$

Equation (23) indicates that the temperature of the modified black holes is different for probes with different energy. Now following the strategy in [30] we propose to define an intrinsic temperature for large modified black holes by identifying probes with radiation particles in the vicinity of black hole horizon. Suppose we make the measurement with the use of radiation photons with average energy $E=<E>$, then we expect that the temperature of black holes can be identified with the energy of photons emitted from black hole $[30,31,39,40,41]$, namely $T=E$. This identification provides a scheme to define an intrinsic temperature for modified black holes. For explicitness, we consider a specific modified dispersion with $f^{2}=1-\left(l_{p} E\right)^{2}$ and $g^{2}=1$. In this case plugging $T=E$ into equation (23) we have

$$
l_{p}^{2} T^{4}-T^{2}+T_{0}^{2}=0
$$


As a result, the intrinsic temperature of modified Schwarzschild (A)dS black holes is obtained as

$$
T_{(A) d S}=\left[\frac{M_{p}^{2}}{2}\left(1-\sqrt{1-\frac{4 T_{0}^{2}}{M_{p}^{2}}}\right)\right]^{\frac{1}{2}} .
$$

Obviously for large black holes with $2 T_{0} \ll M_{p}$, the temperature goes back to the ordinary form $T \sim T_{0}$, while for small black holes with extremely high temperature, we find it reaches to a maximal value $T \sim M_{p} / \sqrt{2}$ as $T_{0} \sim M_{p} / 2$. Correspondingly the radius of the black hole horizon is also bounded from below with a constraint as

$$
\frac{1}{4 \pi}\left(\frac{1}{r_{+}} \pm \frac{3 r_{+}}{L^{2}(0)}\right) \leq M_{p} / 2,
$$

implying $r_{+}>\frac{l_{p}}{2 \pi}$.

The existence of a minimum radius implies that the black hole might stop radiating at the late moment of evaporation. As a matter of fact, this can be seen from the change of the heat capacity of (A)dS Schwarzschild black holes, which is defined as

$$
C_{(A) d S}=\frac{d M}{d T}
$$

From (20) and (26), we obtain it as

$$
C_{(A) d S}=-\frac{2 \pi \sqrt{1-4 l_{p}^{2} T_{0}^{2}}\left(1 \pm \frac{3 r_{+}^{2}}{L^{2}(0)}\right)}{G(0) \sqrt{1-l_{p}^{2} T^{2}}\left(\frac{1}{r_{+}^{2}} \mp \frac{3}{L^{2}(0)}\right)},
$$

where the upper sign and lower sign correspond to AdS and dS black holes respectively.

Thus we find that the heat capacity becomes vanishing when the black hole temperature reaches its maximum value, i.e. $T=\frac{M_{p}}{\sqrt{2}}$. This result implies that there might exist a ground state of modified black holes with non-zero mass such that it may provides a mechanism to take black hole remnants as a candidate for cold dark matter due to their weakly interacting features $[42,43,44]$.

In the remainder of this section we point out that the intrinsic temperature for modified black holes we obtained above, namely equation (23), is consistent with the one for ordinary (A)dS black holes which are supposed to be surrounded by radiation particles endowed with modified dispersion relations. The derivation there can be summarized as follows. Given a modified dispersion relation as (1), we apply it to the photons emitted from ordinary (A)dS 
black holes and identify the characteristic temperature of this black hole with the averaged energy $E$, we may have

$$
T_{(A) d S}^{E U P}=\frac{g(E)}{f(E)} P .
$$

Moreover, we apply an extension of the ordinary uncertainty relation to photons in the vicinity of black hole horizon[45, 46], i.e.,

$$
P \sim \delta P \sim \frac{1}{\delta x}\left(1+\beta^{2} \frac{\delta x^{2}}{L^{2}}\right) \sim \frac{1}{4 \pi r_{+}}\left(1+\beta^{2} \frac{r_{+}^{2}}{L^{2}}\right),
$$

where a "calibration factor" $4 \pi$ as well as a dimensionless parameter $\beta$ is introduced. Plugging this relation into (30) and properly setting the value of $\beta$ we easily find the temperature of (A)dS Schwarzschild black holes has the same form as (23). For details we refer to Ref.[38]. Here we stress that this consistency is universal and independent of the specific form of dispersion relations. Therefore, our results obtained here in the context of modified (A)dS black holes may be viewed as a powerful support for the proposal of deformed equivalence principle in $[22]$.

\section{SUMMARY}

In this paper we investigated (A)dS Schwarzschild black holes in the framework of rainbow gravity with a cosmology constant. We firstly derived a modified (A)dS Schwarzschild black hole solution to the modified vacuum field equation, then studied their thermodynamical properties. In general the temperature of the modified black holes can be obtained by calculating the surface gravity on the horizon. It turns out that it depends on the energy of probes but goes back to the standard result for ordinary (A)dS Schwarzschild black holes as the energy is much less than the Planck energy. When identifying probes as those photons surrounding the horizon of black holes, we also introduced a notion of intrinsic temperature for these modified black holes. In particular, we considered the thermodynamics of a special sort of rainbow black holes by specifying the functions $f(E)$ and $g(E)$. Through our investigations the following two important conclusions may be drawn. First our results for modified (A)dS black holes coincide with those for ordinary (A)dS Schwarzschild black holes, where the temperature is obtained by employing modified dispersion relation and extended uncertainty principle to photons directly. This equivalence testifies the proposal of deformed equivalent principle, namely freely falling observers in rainbow spacetime have the same physics laws as those in doubly special relativity. Second, The modification of (A)dS 
Schwarzschild black holes may provide a reasonable scenario for understanding the late fate of black hole evaporation because in this formalism the evaporation may stop as the heat capacity vanishes. At that moment the temperature of black holes is extremely high, but not divergent as occurring in the ordinary picture of Hawking radiation. Thus the black hole radiation would stop with a remnant which may be viewed as a candidate for mysterious dark matter.

Finally it is worthwhile to point out that our derivations are mainly in a heuristic manner, particularly in the part of the thermodynamics of modified $A d S$ black holes. Firstly we have assumed that the temperature of modified black holes can still be related to the surface gravity as usual, and the reasons that we can do so have been given in[30]. To strictly derive or prove this relation one need a quantum field theory on rainbow spacetime, which as far as we know is still absent, though some relevant work can be found, for instance in Ref.[29]. Secondly, through the paper we identify the expectation value of the energy as the temperature of black holes, which is a standard relation in statistics for radiation particles. However, MDR may also change this relation into $E \sim T\left(1+\delta l p^{2} T^{2}\right)$ with other correction terms $[32,47]$. Therefore, more exact results of the Hawking temperature maybe have to take these modifications into account. However, a delicate calculation shows that this modification will not change the main conclusions we present above at all.

\section{Acknowledgments}

We would like to thank all members, specially Xiang Li and Qingzhang Wu, of the Center for Gravity and Relativistic Astrophysics at Nanchang University for helpful discussions. This work is partly supported by NSFC(Nos.10405027, 10663001), JiangXi SF(Nos. 0612036, 0612038), the key project of Chinese Ministry of Education (No. 208072) and Fok Ying Tung Eduaction Foundation(No.111008). We also acknowledge the support by the Program for Innovative Research Team of Nanchang University.

[1] G. Amelino-Camelia, Int. J. Mod. Phys. D 11, 35 (2002) [arXiv:gr-qc/0012051].

[2] J. Magueijo and L. Smolin, Phys. Rev. Lett. 88, 190403 (2002) [arXiv:hep-th/0112090].

[3] J. Magueijo and L. Smolin, Phys. Rev. D 67, 044017 (2003) [arXiv:gr-qc/0207085]. 
[4] G. Amelino-Camelia, J. Kowalski-Glikman, G. Mandanici and A. Procaccini, Int. J. Mod. Phys. A 20, 6007 (2005) [arXiv:gr-qc/0312124].

[5] F. Girelli, E. R. Livine and D. Oriti, Nucl. Phys. B 708, 411 (2005) [arXiv:gr-qc/0406100].

[6] G. Amelino-Camelia, arXiv:0806.0339 [gr-qc].

G. Amelino-Camelia, arXiv:gr-qc/0309054.

[7] J. Kowalski-Glikman, Lect. Notes Phys. 669, 131 (2005) [arXiv:hep-th/0405273].

[8] L. Smolin, Nucl. Phys. B 742, 142 (2006) [arXiv:hep-th/0501091].

[9] S. Liberati, PoS P2GC, 018 (2007) [arXiv:0706.0142 [gr-qc]].

[10] D. Colladay and V. A. Kostelecky, Phys. Rev. D 58, 116002 (1998) [arXiv:hep-ph/9809521].

[11] S. R. Coleman and S. L. Glashow, Phys. Rev. D 59, 116008 (1999) [arXiv:hep-ph/9812418].

[12] G. Amelino-Camelia and T. Piran, Phys. Rev. D 64, 036005 (2001) [arXiv:astro-ph/0008107].

[13] T. Jacobson, S. Liberati and D. Mattingly, Phys. Rev. D 66, 081302 (2002) [arXiv:hep$\mathrm{ph} / 0112207]$.

[14] R. C. Myers and M. Pospelov, Phys. Rev. Lett. 90, 211601 (2003) [arXiv:hep-ph/0301124].

[15] T. A. Jacobson, S. Liberati, D. Mattingly and F. W. Stecker, Phys. Rev. Lett. 93, 021101 (2004) [arXiv:astro-ph/0309681].

[16] R. Abbasi et al. [HiRes Collaboration], Phys. Rev. Lett. 100, 101101 (2008) [arXiv:astroph/0703099].

[17] G. Amelino-Camelia, M. Arzano, Y. Ling and G. Mandanici, Class. Quant. Grav. 23, 2585 (2006) [arXiv:gr-qc/0506110].

[18] Y. Ling, B. Hu and X. Li, Phys. Rev. D 73, 087702 (2006) [arXiv:gr-qc/0512083].

[19] J. W. Moffat, Int. J. Mod. Phys. D 2, 351 (1993) [arXiv:gr-qc/9211020].

[20] A. Albrecht and J. Magueijo, Phys. Rev. D 59, 043516 (1999) [arXiv:astro-ph/9811018].

[21] L. Mersini-Houghton, M. Bastero-Gil and P. Kanti, Phys. Rev. D 64, 043508 (2001) [arXiv:hep-ph/0101210].

[22] J. Magueijo and L. Smolin, Class. Quant. Grav. 21, 1725 (2004) [arXiv:gr-qc/0305055].

[23] S. Hossenfelder, Phys. Lett. B 649, 310 (2007) [arXiv:gr-qc/0612167].

[24] M. Reuter, Phys. Rev. D 57, 971 (1998) [arXiv:hep-th/9605030].

[25] F. Girelli, S. Liberati, R. Percacci and C. Rahmede, Class. Quant. Grav. 24, 3995 (2007) [arXiv:gr-qc/0607030].

[26] P. Galan and G. A. Mena Marugan, Phys. Rev. D 70, 124003 (2004) [arXiv:gr-qc/0411089]. 
[27] P. Galan and G. A. Mena Marugan, Phys. Rev. D 72, 044019 (2005) [arXiv:gr-qc/0507098].

[28] J. Hackett, Class. Quant. Grav. 23, 3833 (2006) [arXiv:gr-qc/0509103].

[29] R. Aloisio, A. Galante, A. Grillo, S. Liberati, E. Luzio and F. Mendez, Phys. Rev. D 73, 045020 (2006) [arXiv:gr-qc/0511031].

[30] Y. Ling, X. Li and H. Zhang, Mod. Phys. Lett. A 22, 2749 (2007) [arXiv:gr-qc/0512084].

[31] P. Galan and G. A. M. Marugan, Phys. Rev. D 74, 044035 (2006) [arXiv:gr-qc/0608061].

[32] Y. Ling, JCAP 0708, 017 (2007) [arXiv:gr-qc/0609129].

[33] Y. Ling, S. He and H. Zhang, Mod. Phys. Lett. A 22, 2931 (2007) [arXiv:gr-qc/0609130].

[34] F. Girelli, S. Liberati and L. Sindoni, Phys. Rev. D 75, 064015 (2007) [arXiv:gr-qc/0611024].

[35] C. Leiva, J. Saavedra and J. Villanueva, arXiv:0808.2601 [gr-qc].

[36] A. F. Grillo, E. Luzio and F. Mendez, Phys. Rev. D 77, 104033 (2008) [arXiv:0808.2259 [gr-qc]].

[37] J. J. Peng and S. Q. Wu, Gen. Rel. Grav. 40, 2619 (2008) [arXiv:0709.0167 [hep-th]].

[38] X. Han, H. Li and Y. Ling, Phys. Lett. B 666, 121 (2008) [arXiv:gr-qc/0807.4269].

[39] R. J. Adler, P. Chen and D. I. Santiago, Gen. Rel. Grav. 33, 2101 (2001) [arXiv:gr-qc/0106080].

[40] P. Chen and R. J. Adler, Nucl. Phys. Proc. Suppl. 124, 103 (2003) [arXiv:gr-qc/0205106].

[41] F. Scardigli, arXiv:gr-qc/0607010.

[42] J. H. MacGibbon, Nature 329, 308 (1987).

[43] J. D. Barrow, E. J. Copeland and A. R. Liddle, Phys. Rev. D 46, 645 (1992).

[44] B. J. Carr, J. H. Gilbert and J. E. Lidsey, Phys. Rev. D 50, 4853 (1994) [arXiv:astroph/9405027].

[45] B. Bolen and M. Cavaglia, Gen. Rel. Grav. 37, 1255 (2005) [arXiv:gr-qc/0411086].

[46] M. Park, Phys. Lett. B 659, 698 (2008) [arXiv:0709. 2307 [hep-th]].

[47] S. Alexander and J. Magueijo, arXiv:hep-th/0104093.

[48] For recent progress and discussion on this issue, we may refer to [23]. 